\title{
Experimental realization of a topological crystalline insulator in $\mathrm{SnTe}$
}

\author{
Y. Tanaka ${ }^{1}$, Zhi Ren ${ }^{2}$, T. Sato ${ }^{1 \star}$, K. Nakayama ${ }^{1}$, S. Souma ${ }^{3}$, T. Takahashi ${ }^{1,3}$, Kouji Segawa ${ }^{2}$ \\ and Yoichi Ando ${ }^{2 \star}$
}

\begin{abstract}
A topological insulator is an unusual quantum state of matter, characterized by the appearance, at its edges or on its surface, of a gapless metallic state that is protected by time-reversal symmetry ${ }^{1,2}$. The discovery of topological insulators has stimulated the search for other topological states protected by other symmetries ${ }^{3-7}$, such as the recently predicted $^{8}$ topological crystalline insulator $(\mathrm{TCI})$ in which the metallic surface states are protected by the mirror symmetry of the crystal. Here we present experimental evidence for the $\mathrm{TCl}$ phase in tin telluride (SnTe), which has been predicted to be a TCI (ref. 9). Our angle-resolved photoemission spectra show the signature of a metallic Dirac-cone surface band, with its Dirac point slightly away from the edge of the surface Brillouin zone in SnTe. Such a gapless surface state is absent in a cousin material, lead telluride, in line with the theoretical prediction.
\end{abstract}

The surface state of three-dimensional (3D) topological insulators is characterized by a spin non-degenerate Dirac-cone energy dispersion protected by time-reversal symmetry. In topological insulators, the time-reversal symmetry plays a key role in characterizing the topological properties such as the quantum spin Hall effect, the dissipation-less spin current and the magnetoelectric effect $^{10-13}$. In contrast, in TCIs, the metallic surface states are protected by the mirror symmetry (reflection symmetry) of the crystal $^{8}$. The TCIs are characterized by a new topological invariant called the mirror Chern number, instead of the $Z_{2}$ invariant in topological insulators. Intuitively, the existence of mirror symmetry allows one to divide the Hilbert space into left and right, and considering only one of them can single out a non-trivial topology that is otherwise cancelled and undetected. Therefore, even when an insulator is trivial in the topological insulator sense, it can still be non-trivial in the TCI sense when it possesses a mirror symmetry and its mirror Chern number is non-zero ${ }^{8,9}$. Indeed, it was recently shown ${ }^{9}$ that such a situation is realized in an insulating crystal having the rock-salt structure (in which the $\{001\},\{110\}$ and $\{111\}$ surfaces have mirror symmetry with respect to the (110) mirror plane, see Fig. 1a) when a band inversion occurs at the high-symmetry $\mathrm{L}$ points of the bulk Brillouin zone; intriguingly, it was further predicted ${ }^{9}$, on the basis of tight-binding calculations, that a narrow-gap IV-VI semiconductor $\mathrm{SnTe}$ is such a TCI, whereas the isostructural $\mathrm{PbTe}$ is not. It is thus of particular importance to experimentally examine the possibility of the TCI phase in these semiconductors, to establish the concept of this new topological state of matter and possibly to find topological phenomena beyond the framework of known topological materials.
In our angle-resolved photoemission spectroscopy (ARPES) experiment, we paid particular attention to the momentum space around the $\overline{\mathrm{X}}$ point of the surface Brillouin zone corresponding to a projection of the $\mathrm{L}$ point of the bulk Brillouin zone where a direct bulk bandgap resides ${ }^{14-16}$ and the appearance of topological surface states is predicted ${ }^{9}$; note that the (110) mirror plane is projected to the $\bar{\Gamma} \bar{X}$ high-symmetry line in the surface Brillouin zone (Fig. 1a). Our extensive ARPES measurements of the occupied states suggest that the bulk-band maximum is indeed located around the $\bar{X}$ point (see Supplementary Fig. S1). As shown in Fig. 1b, the ARPES intensity at the Fermi level $\left(E_{\mathrm{F}}\right)$ measured with the photon energy $h v=21.2 \mathrm{eV}$ on the (001) surface exhibits a bright intensity pattern centred around the $\bar{X}$ point and is elongated along the $\bar{\Gamma} \overline{\mathrm{X}}$ direction. The band dispersion along two selected cuts (red arrows in Fig. 1b) exhibits a linearly dispersive feature crossing $E_{\mathrm{F}}$, as shown in Fig. 1c,d. The top of this Dirac-like band is located not at the $\overline{\mathrm{X}}$ point but at a point slightly away from it (called here the $\bar{\Lambda}$ point), as one can infer from the band dispersion along the $\bar{\Gamma} \overline{\mathrm{X}}$ cut (Fig. 1e,f) showing the band maxima on both sides of the $\bar{X}$ point $\left(\bar{\Lambda}_{1}\right.$ and $\bar{\Lambda}_{2}$ for the first and second surface Brillouin zones, respectively). Such a characteristic M-shaped dispersion is not expected from the bulk-band calculations at any $k_{z}$ (wave vector perpendicular to the surface) values ${ }^{14-16}$, but is predicted for the surface band $^{9}$ (Supplementary Information), suggesting that the observed Dirac-like band originates from the surface states.

To further examine whether the Dirac-like band is of surface or bulk origin, we have carried out an ARPES measurement along the cut crossing the $\bar{\Lambda}$ point for various photon energies. As one immediately recognizes in Fig. 1g-j, the energy position of the band is stationary with respect to the $h v$ variation. In fact, when we plot the extracted dispersions for different photon energies in the same panel, they overlap each other within the experimental uncertainties of $\sim 0.05 \mathrm{eV}$ (near $E_{\mathrm{F}}$ ) to $\sim 0.1 \mathrm{eV}$ (at higher binding energy, $E_{\mathrm{B}}$ ), demonstrating the surface origin of the Dirac-like band. Note that the broadening of the spectra on the right-hand side of the branch at higher $E_{\mathrm{B}}$ evident in Fig. $1 \mathrm{~h}-\mathrm{j}$ is probably due to a mixture of the bulk state whose energy position changes with $h v$, although the bulk state is obviously very broad and we could not clearly resolve its dispersion.

We note that, because as-grown crystals of SnTe tend to show a heavily hole-doped nature ${ }^{16-18}$, a key to the present observation of the Dirac-like band was to reduce hole carriers in the crystal by minimizing $\mathrm{Sn}$ vacancies during the growth procedure. In fact, the Dirac-like surface state was not resolved in the previous ARPES study ${ }^{16}$, mainly owing to the heavily hole-doped nature

${ }^{1}$ Department of Physics, Tohoku University, Sendai 980-8578, Japan, ${ }^{2}$ Institute of Scientific and Industrial Research, Osaka University, Ibaraki, Osaka 567-0047, Japan, ${ }^{3}$ WPI Research Center, Advanced Institute for Materials Research, Tohoku University, Sendai 980-8577, Japan.

*e-mail: t-sato@arpes.phys.tohoku.ac.jp; y_ando@sanken.osaka-u.ac.jp. 




b

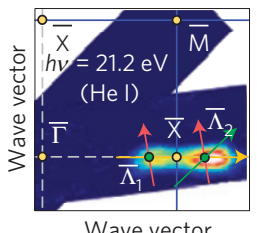

c



d

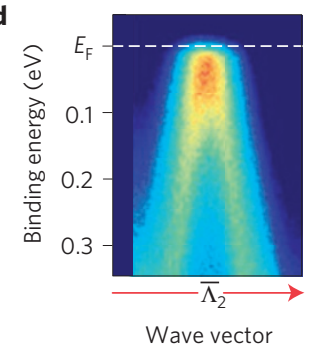

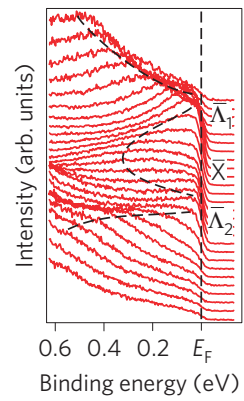

f

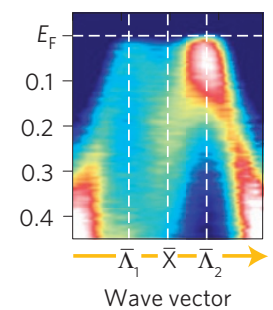

g

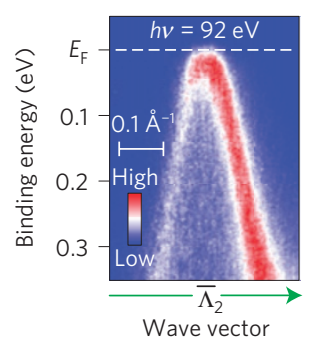

j

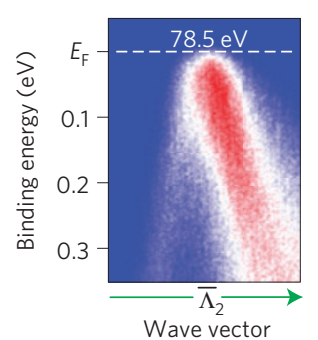

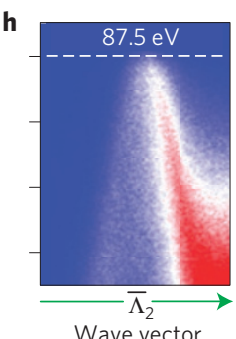

Wave vector

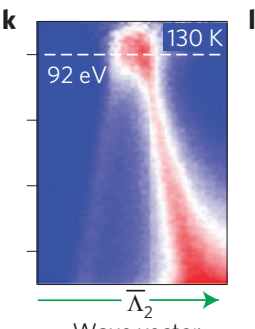

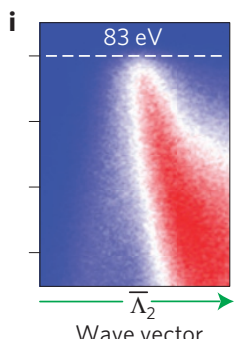

Wave vector

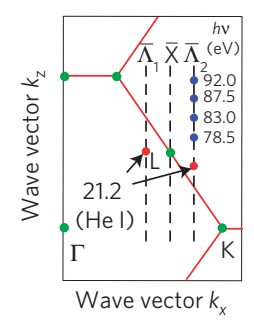

m

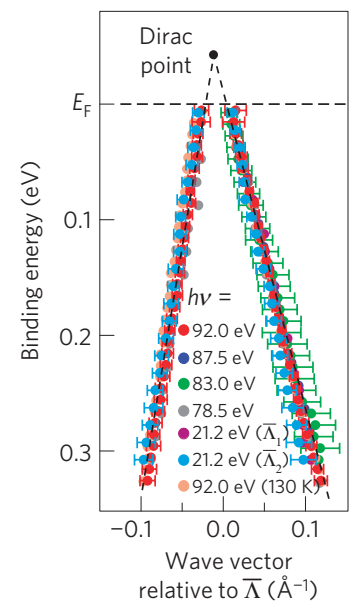

Figure 1 | Dirac-like band dispersion in SnTe. a, The bulk Brillouin zone (red lines) and the corresponding (001) surface Brillouin zone (blue lines). The (110) mirror plane is indicated by the green shaded area. $\mathbf{b}$, ARPES intensity mapping at $E_{\mathrm{F}}$ at $T=30 \mathrm{~K}$ for $\mathrm{SnTe}$ plotted as a function of the $2 \mathrm{D}$ wave vector measured with the $\mathrm{He} 1$ line ( $h v=21.2 \mathrm{eV}$ ); this intensity is obtained by integrating the spectra within \pm 10 meV of $E_{\mathrm{F}}$. c,d, Near- $E_{\mathrm{F}}$ ARPES intensity measured at $h v=21.2 \mathrm{eV}$ as a function of the wave vector and $E_{\mathrm{B}}$ along the cut crossing the $\bar{\Lambda}_{1}$ and $\bar{\Lambda}_{2}$ point (red arrows in $\mathbf{b}$ ), respectively. e,f, Energy distribution curves (e) along the $\bar{\Gamma} \bar{X}$ cut (yellow arrow in $\mathbf{b}$ ) measured at $h v=21.2 \mathrm{eV}$, and the corresponding intensity plot (f). The dashed lines in $\mathbf{e}$ are a guide to the eyes to trace the band dispersion. $\mathbf{g}-\mathbf{j}$, ARPES intensity measured at $T=30 \mathrm{~K}$ with various photon energies across the cut crossing the $\bar{\Lambda}_{2}$

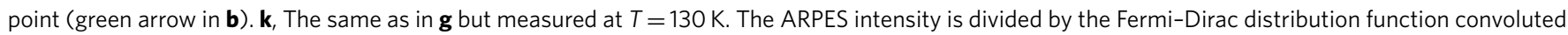

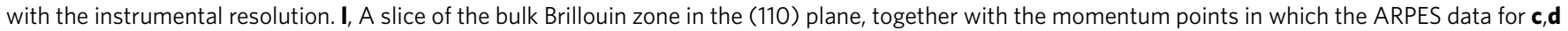
and $\mathbf{g}$-k were obtained; $k_{z}$ values were estimated by using the inner-potential value of $8.5 \mathrm{eV}$ as determined by the normal-emission ARPES measurement.


the green arrow in $\mathbf{b}$; error bars are shown for the data at $h v=92,83$, and $21.2 \mathrm{eV}$, and they reflect the uncertainties originating from the momentum resolution and the standard deviation in the peak positions of momentum distribution curves. The $h v=83 \mathrm{eV}$ data are particularly broad at high $E_{\mathrm{B}}$, which is partly due to a mixing of the bulk band.

of the sample (chemical potential was located $\sim 0.5 \mathrm{eV}$ lower when compared with our data). Furthermore, a downward band bending, possibly due to a loss of Te atoms on cleaving, was obviously taking place near the surface (Supplementary Information), which further worked in our favour.

As shown in Fig. 1k, the ARPES data at $T=130 \mathrm{~K}$ divided by the Fermi-Dirac distribution function indicate that the leftand right-hand side dispersion branches actually merge into a single peak above $E_{\mathrm{F}}$. The Dirac-point energy is estimated to be $0.05 \mathrm{eV}$ above $E_{\mathrm{F}}$ from a linear extrapolation of the two dispersion branches (Fig. 1m) that were determined from the peak positions in the momentum distribution curves; furthermore, the Dirac band velocities extracted from the dispersions are 4.5 and $3.0 \mathrm{eV} \AA$, for the left- and right-hand side branches, respectively. One can see in Fig. $1 \mathrm{~m}$ that the band dispersion exhibits no discernible change with temperature (compare the $30 \mathrm{~K}$ and $130 \mathrm{~K}$ data for $h v=92 \mathrm{eV}$ ). 

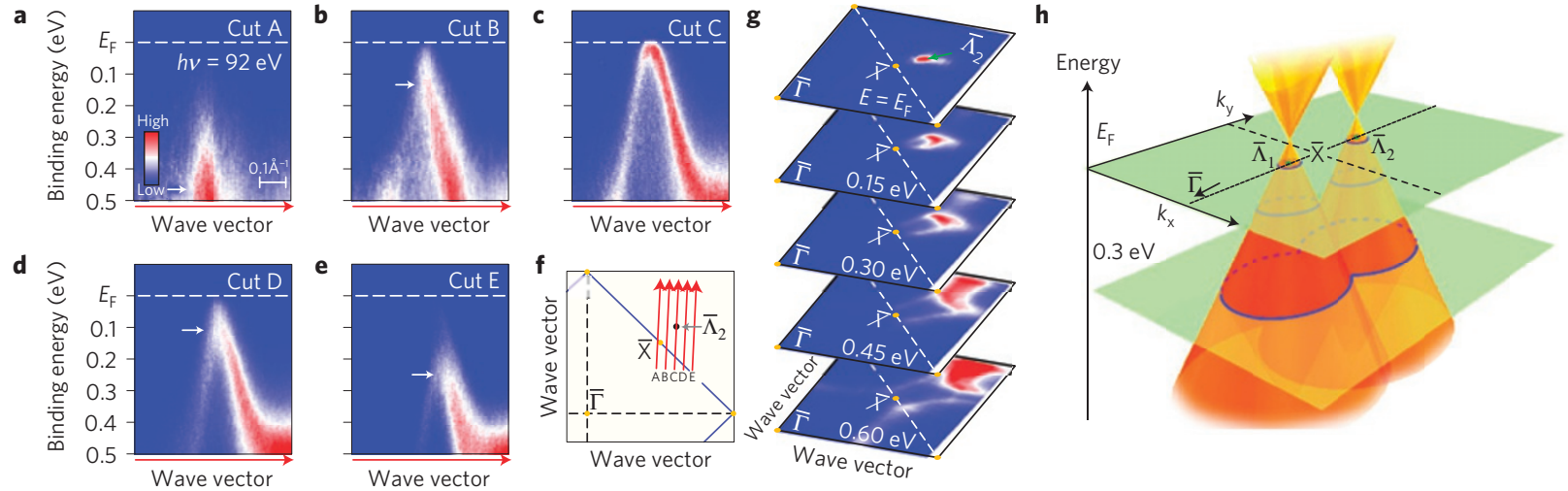

Figure 2 | Two-dimensional band dispersion of SnTe. a-e, Near- $E_{\mathrm{F}}$ ARPES intensity for SnTe as a function of the wave vector and $E_{\mathrm{B}}$ measured at $h \nu=92 \mathrm{eV}$ along the cuts (A-E) around the $\bar{\Lambda}_{2}$ point shown by the red arrows in the surface Brillouin zone in $\mathbf{f}$. The white arrow marks the top of the dispersion. f, Surface Brillouin zone and the momentum cuts of the measurement (red arrows). $\mathbf{g}$, ARPES intensity mappings for SnTe in a 2D wave-vector plane at various $E_{\mathrm{B}}$ values. Note that the intensity distribution for $E_{\mathrm{B}} \geq 0.45 \mathrm{eV}$ is strongly affected by the bulk-band contributions. $\mathbf{h}$, Schematic $2 \mathrm{D}$ band dispersions near $E_{\mathrm{F}}$ depicting the evolution of the surface Dirac cones. The blue circles show energy contours for representative $E_{\mathrm{B}}$ slices, highlighting the Lifshitz transition ${ }^{9}$ (see Supplementary Information for details).
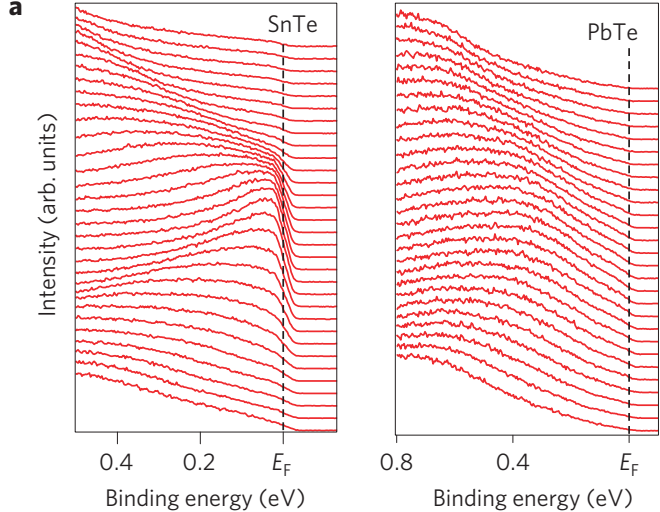

b
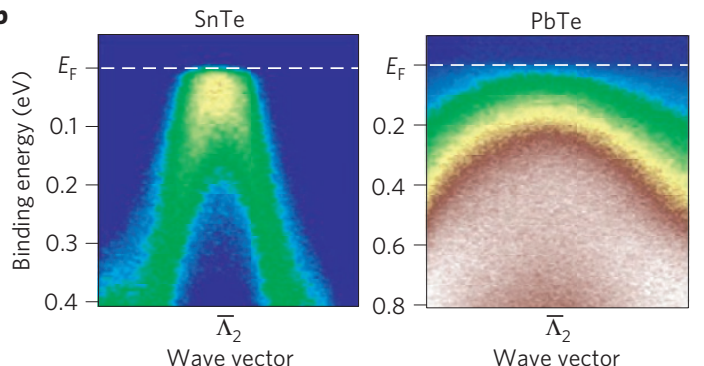

c



Figure 3 | Comparison of the band structure between $\mathrm{SnTe}$ and $\mathrm{PbTe}$. a, Near- $E_{\mathrm{F}}$ energy distribution curves around the $\bar{\Lambda}$ point for $\mathrm{SnTe}$ and $\mathrm{PbTe}$ measured with $h v=21.2 \mathrm{eV}$; note that the energy axes are different for SnTe and PbTe. b, Corresponding ARPES-intensity plots. c, Schematic illustration of the evolution of the band dispersion in $\mathrm{Pb}_{1-x} \mathrm{Sn}_{x} \mathrm{Te}$ expected from the present ARPES experiment. SS, CB, and VB denote the surface state, the bulk conduction band and the bulk valence band, respectively.
One may argue that in SnTe the well-known rhombohedral distortion ${ }^{19}$ would break the mirror symmetry with respect to the (110) plane and destroy the signature of the Dirac-cone surface states. However, one can safely exclude this possibility in the present experiment, because the rhombohedral phase transition temperature is known to be strongly dependent on the carrier density $^{19}$ and in our samples the transition occurs well below $30 \mathrm{~K}$ (the temperature of our ARPES measurements), which is corroborated by the absence of a kink in the temperature dependence of the resistivity (see Supplementary Fig. S6). In addition, the (001) surface of the cubic structure has two mirror planes, and the rhombohedral distortion breaks the mirror symmetry of only one of those two mirror planes. This means that two of the original four Dirac cones remain gapless in the rhombohedral phase; furthermore, as the rhombohedral distortion in SnTe is weak (it induces only $1.6 \%$ displacement of the atomic positions ${ }^{19}$ ), the expected result is an opening of a small gap at the Dirac point in the other two Dirac cones. Therefore, the small rhombohedral distortion, even when it happens, will not significantly change the surface state spectrum.

To further elucidate the topology of the Dirac cone in detail, we have determined the whole band dispersion in the $2 \mathrm{D}$ momentum space. By selecting a specific photon energy $(h v=92 \mathrm{eV})$, owing to the matrix-element effects, we found it possible to pick up the dispersion of a single Dirac cone centred at the $\bar{\Lambda}_{2}$ point $\left(\bar{\Lambda}_{2}\right.$ Dirac cone) while suppressing the intensity of the $\bar{\Lambda}_{1}$ Dirac cone. Figure $2 \mathrm{a}-\mathrm{e}$ shows the near- $E_{\mathrm{F}}$ ARPES intensity measured along several cuts (A-E) around the $\bar{\Lambda}_{2}$ point. Along cut A (Fig. 2a), the surface band has its top at an $E_{\mathrm{B}}$ of $0.45 \mathrm{eV}$. On moving from cut $\mathrm{A}$ to $\mathrm{E}$, the band maximum (white arrow) approaches $E_{\mathrm{F}}$ (cuts $\mathrm{A}-\mathrm{B}$ ), passes $E_{\mathrm{F}}$ (cut $\mathrm{C}$ ), and then disperses back again towards higher $E_{\mathrm{B}}$ (cuts D-E). This result establishes the cone-shaped dispersion of the Dirac-like band in the 2D momentum space as in threedimensional topological insulators ${ }^{1,2}$ and graphene ${ }^{20}$. In passing, we have surveyed electronic states throughout the Brillouin zone and found no evidence for other metallic surface states (Supplementary Fig. S1) and thus conclude that the surface electronic states consist of four Dirac cones in the first surface Brillouin zone. This indicates that this material is not a topological insulator but is a TCI owing to an even number of band-inversion points ${ }^{9}$ that is reflected in the number of Dirac cones.

As shown in the ARPES-intensity contour plots in Fig. $2 \mathrm{~g}$ for several $E_{\mathrm{B}}$ slices, the Dirac cone in SnTe is anisotropic and slightly elongated along the $\bar{\Gamma} \bar{X}$ direction, and its topology shows a Lifshitz 
transition as a function of band filling: namely, at $E_{\mathrm{B}}=0.15 \mathrm{eV}$ the cross-section is closed and is elongated towards $\bar{X}$, but it becomes open at $E_{\mathrm{B}}=0.30 \mathrm{eV}$, suggesting that it is reconnected with the Dirac cone on the other side of $\bar{X}$ (see Supplementary Fig. S5); at $E_{\mathrm{B}} \geq 0.45 \mathrm{eV}$, the bulk band creates strong intensities and partly smears the surface state. This Lifshitz transition is interesting, because it would accompany a marked change in the Dirac-carrier properties and provide another ingredient in the physics of topological insulators. The observed evolution in the $2 \mathrm{D}$ band dispersion presenting a double Dirac-cone structure is schematically depicted in Fig. $2 \mathrm{~h}$. Although SnTe samples are always p-type, one can access the upper part of this double cone by using scanning tunnelling microscopy or field-effect-transistor devices.

To see how unique SnTe is among isostructural IV-VI semiconductors, we have performed an ARPES measurement of PbTe and directly compared the near- $E_{\mathrm{F}}$ electronic states around the $\bar{\Lambda}$ point, as shown in Fig. 3a,b. The PbTe single crystals used here were specially tuned to be only weakly electron doped and were of very high quality, with an electron mobility of $60,000 \mathrm{~cm}^{2} \mathrm{~V}^{-1} \mathrm{~s}^{-1}$ (Supplementary Information). Intriguingly, the spectral feature of PbTe shows no evidence for the metallic Dirac-like band, and exhibits only a broad feature originated from the top of the bulk valence band ${ }^{21}$, suggesting that this material is an ordinary (trivial) insulator. Note that this broad feature is intrinsic and is not due to a bad crystallinity, because deeper bands show clear dispersions (Supplementary Information). This naturally suggests that a topological phase transition takes place in a solid-solution system $\mathrm{Pb}_{1-x} \mathrm{Sn}_{x} \mathrm{Te}$ (Fig. 3c). This conclusion agrees with the tight-binding calculation ${ }^{9}$ that predicted that the valence bands at four $\mathrm{L}$ points in $\mathrm{SnTe}$ are inverted relative to $\mathrm{PbTe}$, resulting in different mirror Chern numbers ( 2 versus 0 ). One can thus infer that the bulk bandgap closes at a critical $x$ value, $x_{c}$, accompanied by a parity change of the valence-band wavefunction and an emergence/disappearance of the Dirac-cone surface state. Therefore, the present results have established the TCI phase in $\mathrm{SnTe}$, which is in contrast to the trivial nature of isostructural $\mathrm{PbTe}$. Our results unambiguously demonstrate the validity of the concept of TCIs and suggests the existence of many more kinds of topological material.

\section{Methods}

High-quality single crystals of SnTe and PbTe were grown by a modified Bridgeman method in sealed evacuated quartz-glass tubes from high-purity elements ( $\mathrm{Sn}$ (99.99\%), Pb (99.998\%), Te (99.999\%)). To obtain SnTe crystals with minimal Sn vacancy, a starting ratio of $\mathrm{Sn} / \mathrm{Te}=51: 49$ was chosen and, after melting the elements at high temperature, the tube was slowly cooled to $770^{\circ} \mathrm{C}$ (which is only $20^{\circ} \mathrm{C}$ below the melting point) and quenched into cold water; the carrier density estimated from the Hall coefficient was $2 \times 10^{20} \mathrm{~cm}^{-3}$, which is lower than usual ${ }^{17,18}$. PbTe crystals were grown with a starting composition of $\mathrm{Pb} / \mathrm{Te}=1.005: 1$ by slowly cooling the melt from $980^{\circ} \mathrm{C}$ to $700{ }^{\circ} \mathrm{C}$ at $2^{\circ} \mathrm{Ch}^{-1}$ and holding at $700{ }^{\circ} \mathrm{C}$ for $12 \mathrm{~h}$; the carrier density in the resulting crystals was very low at $1.7 \times 10^{17} \mathrm{~cm}^{-3}$. Both $\mathrm{SnTe}$ and $\mathrm{PbTe}$ single crystals are single domain and show good crystallinity in X-ray Laue analysis, and their detailed transport properties are described in the Supplementary Information. ARPES measurements were performed with the MBS-A1 and VG-Scienta SES2002 electron analysers with a high-intensity helium discharge lamp at Tohoku University and also with tunable synchrotron lights at the beamline BL28A at the Photon Factory (KEK). To excite photoelectrons, we used the $\mathrm{He} \mathrm{I} \alpha$ resonance line $(h \nu=21.218 \mathrm{eV})$ and the circularly polarized lights of $50-100 \mathrm{eV}$ at Tohoku University and the Photon Factory, respectively. The energy and angular resolutions were set at $10-30 \mathrm{meV}$ and $0.2^{\circ}$, respectively. Samples were cleaved in situ along the (001) crystal plane in an ultrahigh vacuum of $1 \times 10^{-10}$ torr at room temperature. A shiny mirror-like surface was obtained after cleaving the samples, confirming their high quality. The Fermi level of the samples was referenced to that of a gold film evaporated onto the sample holder.
Received 23 June 2012; accepted 7 September 2012; published online 30 September 2012

\section{References}

1. Hasan, M. Z. \& Kane, C. L. Colloquium: Topological insulators. Rev. Mod. Phys. 82, 3045-3067 (2010).

2. Qi, X-L. \& Zhang, S-C. Topological insulators and superconductors. Rev. Mod. Phys. 83, 1057-1110 (2011).

3. Schnyder, A. P., Ryu, S., Furusaki, A. \& Ludwig, A. W. W. Classification of topological insulators in three spatial dimensions. Phys. Rev. B 78, 195125 (2008).

4. Kitaev, A. Periodic table for topological insulators and superconductors. Preprint at http://arxiv.org/abs/0901.2686v2 (2009).

5. Ran, Y. Weak indices and dislocations in general topological band structures. Preprint at http://arxiv.org/abs/1006.5454v2 (2010).

6. Mong, R. S. K., Essin, A. M. \& Moore, J. E. Antiferromagnetic topological insulators. Phys. Rev. B 81, 245209 (2010).

7. Li, R., Wang, J., Qi, X-L. \& Zhang, S-C. Dynamical axion field in topological magnetic insulators. Nature Phys. 6, 284-288 (2010).

8. Fu, L. Topological crystalline insulators. Phys. Rev. Lett. 106, 106802 (2011).

9. Hsieh, T. H. et al. Topological crystalline insulators in the SnTe material class. Nature Commun. 3, 982 (2012).

10. Kane, C. L. \& Mele, E. J. $Z_{2}$ topological order and the quantum spin Hall effect. Phys. Rev. Lett. 95, 146802 (2005).

11. Bernevig, B. A., Hughes, T. L. \& Zhang, S-C. Quantum spin Hall effect and topological phase transition in HgTe quantum wells. Science 314, $1757-1761$ (2006)

12. Xu, C. \& Moore, J. E. Stability of the quantum spin Hall effect: Effects of interactions, disorder, and $Z_{2}$ topology. Phys. Rev. B 73, 045322 (2006).

13. Qi, X-L., Hughes, T. L. \& Zhang, S-C. Topological field theory of time-reversal invariant insulators. Phys. Rev. B 78, 195424 (2008).

14. Tung, Y. W. \& Cohen, M. L. Relativistic band structure and electronic properties of SnTe, GeTe, and PbTe. Phys. Rev. 180, 823-826 (1969).

15. Melvin, J. S. \& Hendry, D. C. Self-consistent relativistic energy bands for tin telluride. J. Phys. C 12, 3003-3012 (1979).

16. Littlewood, P. B. et al. Band structure of SnTe studied by photoemission spectroscopy. Phys. Rev. Lett. 105, 086404 (2010).

17. Richard Burke, J. Jr, Allgaier, R. S., Houston, B. B., Babiskin, J. \& Siebenmann, P. G. Shubnikov-de Haas effect in SnTe. Phys. Rev. Lett. 14, 360-361 (1965).

18. Allgaier, R. S. \& Houston, B. Weak-field magnetoresistance and the valence-band structure of SnTe. Phys. Rev. B 5, 2186-2197 (1972).

19. Iizumi, M., Hamaguchi, Y., Komatsubara, K. F. \& Kato, Y. Phase transition in SnTe with low carrier concentration. J. Phys. Soc. Jpn 38, 443-449 (1975).

20. Bostwick, A., Ohta, T., Seyller, T., Horn, K. \& Rotenberg, E. Quasiparticle dynamics in graphene. Nature Phys. 3, 36-40 (2007).

21. Nakayama, K., Sato, T., Takahashi, T. \& Murakami, H. Doping induced evolution of Fermi surface in low carrier superconductor Tl-doped PbTe. Phys. Rev. Lett. 100, 227004 (2008).

\section{Acknowledgements}

We thank L. Fu for stimulating discussions. We also thank M. Komatsu, M. Nomura, E. Ieki, T. Takahashi, N. Inami, H. Kumigashira and K. Ono for their assistance in ARPES measurements, and T. Ueyama and K. Eto for their assistance in crystal growth. This work was supported by JSPS (NEXT Program and KAKENHI 23224010), JST-CREST, MEXT of Japan (Innovative Area Topological Quantum Phenomena), AFOSR (AOARD 124038 ) and KEK-PF (proposal number: 2012S2-001).

\section{Author contributions}

Y.T., T.S., K.N., S.S. and T.T. performed ARPES measurements. Z.R., K.S. and Y.A. carried out the growth of the single crystals and their characterizations. Y.T., T.S. and Y.A. conceived the experiments and wrote the manuscript.

\section{Additional information}

Supplementary information is available in the online version of the paper. Reprints and permissions information is available online at www.nature.com/reprints. Correspondence and requests for materials should be addressed to T.S. or Y.A.

\section{Competing financial interests}

The authors declare no competing financial interests. 